\title{
A DETAILED ANALYSIS OF THE HD 73526 2:1 RESONANT PLANETARY SYSTEM*
}

\author{
Robert A. Wittenmyer ${ }^{1,2}$, Xianyu Tan ${ }^{3,4}$, Man Hoi LeE ${ }^{3,5}$, Jonathan Horner ${ }^{1,2}$, C. G. Tinney ${ }^{1,2}$, R. P. Butler ${ }^{6}$, \\ G. S. SAlter ${ }^{1,2}$, B. D. Carter ${ }^{7}$, H. R. A. Jones ${ }^{8}$, S. J. O’Toole ${ }^{9}$, J. Bailey ${ }^{1,2}$, D. Wright ${ }^{1,2}$, J. D. Crane ${ }^{10}$, \\ S. A. Schectman ${ }^{10}$, P. Arriagada ${ }^{6}$, I. Thompson ${ }^{10}$, D. Minniti ${ }^{11,12}$, and M. Diaz ${ }^{11}$ \\ ${ }^{1}$ School of Physics, University of New South Wales, Sydney 2052, Australia; rob@ phys.unsw.edu.au \\ ${ }^{2}$ Australian Centre for Astrobiology, University of New South Wales, Sydney 2052, Australia \\ ${ }^{3}$ Department of Earth Sciences, The University of Hong Kong, Pokfulam Road, Hong Kong \\ ${ }^{4}$ Department of Planetary Sciences and Lunar and Planetary Laboratory, The University of Arizona, \\ 1629 University Boulevard, Tucson, AZ 85721, USA \\ ${ }^{5}$ Department of Physics, The University of Hong Kong, Pokfulam Road, Hong Kong \\ ${ }^{6}$ Department of Terrestrial Magnetism, Carnegie Institution of Washington, \\ 5241 Broad Branch Road, NW, Washington, DC 20015-1305, USA \\ ${ }^{7}$ Faculty of Sciences, University of Southern Queensland, Toowoomba, Queensland 4350, Australia \\ ${ }^{8}$ University of Hertfordshire, Centre for Astrophysics Research, Science and Technology Research Institute, \\ College Lane, AL10 9AB, Hatfield, UK \\ ${ }^{9}$ Australian Astronomical Observatory, PO Box 915, North Ryde, NSW 1670, Australia \\ ${ }^{10}$ The Observatories of the Carnegie Institution of Washington, 813 Santa Barbara Street, Pasadena, CA 91101, USA \\ ${ }^{11}$ Institute of Astrophysics, Pontificia Universidad Catolica de Chile, Casilla 306, Santiago 22, Chile \\ 12 Vatican Observatory, V00120 Vatican City State, Italy \\ Received 2013 August 14; accepted 2013 November 26; published 2013 December 18
}

\begin{abstract}
We present six years of new radial velocity data from the Anglo-Australian and Magellan Telescopes on the HD 73526 2:1 resonant planetary system. We investigate both Keplerian and dynamical (interacting) fits to these data, yielding four possible configurations for the system. The new data now show that both resonance angles are librating, with amplitudes of $40^{\circ}$ and $60^{\circ}$, respectively. We then perform long-term dynamical stability tests to differentiate these solutions, which only differ significantly in the masses of the planets. We show that while there is no clearly preferred system inclination, the dynamical fit with $i=90^{\circ}$ provides the best combination of goodness-of-fit and long-term dynamical stability.
\end{abstract}

Key words: methods: numerical - planets and satellites: dynamical evolution and stability planets and satellites: individual (HD 73526) - techniques: radial velocities

Online-only material: color figures

\section{INTRODUCTION}

The ever-growing population of known multiple-planet systems has proven to be an exceedingly useful laboratory for testing models of planetary system formation and dynamical evolution. Of particular interest are the systems that are in or near resonant configurations. A number of such systems have been identified from radial velocity surveys, with some notable examples including GJ 876 (Marcy et al. 2001), HD 128311 (Vogt et al. 2005), HD 82943 (Mayor et al. 2004), and HD 200964 (Johnson et al. 2011). Wright et al. (2011) noted that about $1 / 3$ of well-characterized multi-planet systems were in such low-order period commensurabilities. The Kepler mission has revealed hundreds of candidate multiply transiting planetary systems (Borucki et al. 2010; Batalha et al. 2013), some of which are in or near low-order resonances (Lissauer et al. 2011; Steffen et al. 2013). One emerging trend from the Kepler results is that a significant number of such near-resonant planet pairs are outside of the resonance (Fabrycky et al. 2012; Veras \& Ford 2012; Lee et al. 2013), with an excess population slightly wide of the resonance and a deficit of planet pairs just inside the resonance (Lithwick \& Wu 2012).

Marti et al. (2013) recently showed that the 4:2:1 Laplace resonance in the GJ 876 system (Rivera et al. 2010; Baluev 2011) acts to stabilize the three outer planets, constraining their mutual inclinations to less than $20^{\circ}$ and $e_{3} \lesssim 0.05$. Tan et al.

\footnotetext{
* This paper includes data gathered with the 6.5 meter Magellan Telescopes located at Las Campanas Observatory, Chile.
}

(2013) applied a dynamical fitting approach to $10 \mathrm{yr}$ of precise Keck radial velocities of the HD 82943 2:1 resonant system (Lee et al. 2006). They found a best fit at an inclination of $20 \pm 4^{\circ}$ to the sky plane, which was dynamically stable despite the high planetary masses implied by that inclination. Interestingly, Herschel debris-disk observations reported by Kennedy et al. (2013) show that the disk has a similar line-of-sight inclination of $27 \pm 4^{\circ}$. These examples show how planetary systems can be characterized with multiple complementary approaches.

HD 73526 is one of 20 stars added to the Anglo-Australian Planet Search (AAPS) in late 1999, based on high metallicity and the then-emerging planet-metallicity correlation (Laughlin 2000; Valenti \& Fischer 2005). The first planet, HD 73526b (Tinney et al. 2003), was reported to have period $P=190.5 \pm 3.0$ days, an eccentricity $e=0.34 \pm 0.08$, and a minimum mass $m \sin i=3.0 \pm 0.3 M_{\text {Jup }}$. Tinney et al. (2006) reported a second planet with $P=376.9 \pm 0.9$ days, placing it in the 2:1 resonance with the inner planet. The authors noted that the $2: 1$ period commensurability appears to be common, with four of the 18 then-known multiple systems moving on such orbits. The HD 73526 planetary system was reported in a 2:1 mean-motion resonance (MMR), with $\theta_{1}$ librating around $0^{\circ}$ and $\theta_{2}$ circulating (Tinney et al. 2006), where $\theta_{1}$ and $\theta_{2}$ are the lowest order, eccentricity-type 2:1 MMR angles:

$$
\begin{aligned}
& \theta_{1}=\lambda_{1}-2 \lambda_{2}+\varpi_{1}, \\
& \theta_{2}=\lambda_{1}-2 \lambda_{2}+\varpi_{2} .
\end{aligned}
$$


Here, $\lambda$ is the mean longitude, $\varpi$ is the longitude of periapse, and the subscripts 1 and 2 represent the inner and outer planets, respectively. This type of 2:1 MMR configuration is dynamically interesting as it cannot be produced by smooth migration capture alone (Beaugé et al. 2003; Ferraz-Mello et al. 2003; Lee 2004; Beaugé et al. 2006; Michtchenko et al. 2008) and alternative mechanisms have been suggested to produce such a configuration.

The resonant property of the HD 73526 planets makes this an interesting system in terms of its dynamical evolution. Subsequent work has focused on how planets get into the 2:1 resonance in this and other exoplanetary systems. Sándor et al. (2007) proposed that the HD 73526 system experienced both migration and a sudden perturbation (planet-planet scattering or rapid dissipation of the protoplanetary disk) that combined to drive the system into the observed 2:1 resonance. Similarly, Zhang et al. (2010) suggested that the HD 73526 and HD 128311 systems, both of which are in 2:1 librating-circulating resonances, arrived in that configuration via a hybrid mechanism of scattering and collisions with terrestrial planetesimals. Scattering into low-order resonances was also implicated by Raymond et al. (2008) as a likely formation mechanism, where scattering events drive the two larger planets into a resonance while ejecting the smaller planet. In summary, there is general agreement that the HD 73526 system did not arrive in the 2:1 resonance by smooth migration alone.

The aim of this work is to provide an updated set of parameters for the HD 73526 system, based on an additional six years of AAPS observations, as well as new data from Magellan (Section 2). In addition, we perform Keplerian and full dynamical fits to the complete dataset (Section 3). In Section 4, we present detailed dynamical stability maps of the system, using both the parameters from the Keplerian and dynamical fits. Finally, in Section 5, we offer conclusions on the architecture of the system based on the combination of our orbit fitting and dynamical stability analysis.

\section{OBSERVATIONS}

\subsection{Anglo-Australian Telescope}

AAPS Doppler measurements are made with the UCLES echelle spectrograph (Diego et al. 1990). An iodine absorption cell provides wavelength calibration from 5000 to $6200 \AA$. The spectrograph point-spread function (PSF) and wavelength calibration are derived from the iodine absorption lines embedded in every pixel of the spectrum by the cell (Valenti et al. 1995; Butler et al. 1996). The result is a precision Doppler velocity estimate for each epoch, along with an internal uncertainty estimate, which includes the effects of photon-counting uncertainties, residual errors in the spectrograph PSF model, and variation in the underlying spectrum between the iodine-free template and the epoch spectra observed through the iodine cell. All velocities are measured relative to the zero-point defined by the template observation. A total of 36 Anglo-Australian Telescope (AAT) observations have been obtained since 1999 February 2 (Table 1) and were used in the following analysis, representing a data span of 4836 days. The exposure times range from 300 to $900 \mathrm{~s}$, and the mean internal velocity uncertainty for these data is $4.1 \mathrm{~m} \mathrm{~s}^{-1}$.

\subsection{Magellan Telescope}

Since HD 73526 is among the faintest AAPS targets $(V=$ 9.0), we have obtained supplemental observations with the
Table 1

AAT/UCLES Radial Velocities for HD 73526

\begin{tabular}{|c|c|c|}
\hline JD-2400000 & $\begin{array}{l}\text { Velocity } \\
\left(\mathrm{m} \mathrm{s}^{-1}\right)\end{array}$ & $\begin{array}{c}\text { Uncertainty } \\
\left(\mathrm{m} \mathrm{s}^{-1}\right)\end{array}$ \\
\hline 51212.13020 & 7.91 & 5.40 \\
\hline 51213.13145 & 0.32 & 5.37 \\
\hline 51214.23895 & 5.52 & 6.54 \\
\hline 51236.14647 & 15.70 & 6.62 \\
\hline 51630.02802 & 3.91 & 5.12 \\
\hline 51717.89996 & -190.60 & 7.09 \\
\hline 51920.14186 & -77.28 & 6.45 \\
\hline 51984.03780 & 10.04 & 4.87 \\
\hline 52009.09759 & 12.38 & 4.21 \\
\hline 52060.88441 & -105.26 & 3.73 \\
\hline 52091.84653 & -223.76 & 6.76 \\
\hline 52386.90032 & -2.62 & 3.46 \\
\hline 52387.89210 & 1.72 & 2.86 \\
\hline 52420.92482 & -66.78 & 3.28 \\
\hline 52421.91992 & -64.87 & 3.23 \\
\hline 52422.86019 & -66.65 & 3.34 \\
\hline 52424.92369 & -77.31 & 7.02 \\
\hline 52454.85242 & -151.57 & 3.44 \\
\hline 52655.15194 & -81.59 & 3.53 \\
\hline 53008.13378 & 0.13 & 2.42 \\
\hline 53045.13567 & -95.56 & 3.20 \\
\hline 53399.16253 & -52.76 & 2.97 \\
\hline 53482.87954 & 20.95 & 2.02 \\
\hline 53483.88740 & 26.55 & 2.59 \\
\hline 53485.96240 & 22.83 & 3.65 \\
\hline 53488.93814 & 14.81 & 2.33 \\
\hline 53506.88650 & 5.03 & 2.25 \\
\hline 53508.91266 & 11.94 & 2.03 \\
\hline 53515.89441 & -4.01 & 2.89 \\
\hline 53520.91025 & -4.97 & 3.27 \\
\hline 54041.18613 & -14.76 & 7.28 \\
\hline 54549.03413 & -97.63 & 2.83 \\
\hline 54899.03133 & -7.35 & 4.24 \\
\hline 55315.92532 & -91.43 & 3.06 \\
\hline 55997.03979 & 62.28 & 4.10 \\
\hline 56048.94441 & -57.19 & 4.16 \\
\hline
\end{tabular}

Planet Finder Spectrograph (PFS; Crane et al. 2006, 2008, 2010 ) on the $6.5 \mathrm{~m}$ Magellan II (Clay) telescope. The PFS is a high-resolution $(R \sim 80,000)$ echelle spectrograph optimized for high-precision, radial velocity measurements (e.g., Albrecht et al. 2011, 2012; Anglada-Escudé et al. 2012; Arriagada et al. 2013). The PFS also uses the iodine cell method, as described above, to obtain precise radial velocities. The 20 measurements of HD 73526 are given in Table 2. The data span 856 days and have a mean internal uncertainty of $1.2 \mathrm{~m} \mathrm{~s}^{-1}$.

\section{ORBIT FITTING}

\subsection{Non-interacting Keplerian Fit}

New radial velocity observations of exoplanetary systems can sometimes result in substantial modification of the best-fit planetary orbits. For example, the two planets in the HD 155358 system were initially reported to be in orbital periods of 195 and 530 days (Cochran et al. 2007). A further five years of observations revealed that the outer planet actually has an orbital period of 391.9 days and is trapped in the 2:1 MMR (Robertson et al. 2012a). In light of the possibility that the best-fit orbits of the two planets may be significantly different than initially presented in Tinney et al. (2006), we begin our orbit fitting process with a wide-ranging search using a genetic algorithm. This approach is often used when the orbital parameters of a 
Table 2

Magellan/PFS Radial Velocities for HD 73526

\begin{tabular}{lrc}
\hline \hline JD-2400000 & $\begin{array}{r}\text { Velocity } \\
\left(\mathrm{m} \mathrm{s}^{-1}\right)\end{array}$ & $\begin{array}{r}\text { Uncertainty } \\
\left(\mathrm{m} \mathrm{s}^{-1}\right)\end{array}$ \\
\hline 55582.79672 & 14.7 & 1.2 \\
55584.75698 & 20.6 & 1.2 \\
55585.74045 & 22.1 & 1.2 \\
55587.77487 & 28.3 & 1.0 \\
55588.71850 & 28.4 & 0.9 \\
55663.53102 & -60.3 & 1.1 \\
55668.54537 & -73.8 & 0.8 \\
55672.50855 & -94.5 & 0.8 \\
55953.76750 & 0.4 & 1.3 \\
55955.71181 & 0.0 & 1.1 \\
56282.77476 & -135.9 & 1.5 \\
56292.76731 & -101.0 & 1.3 \\
56345.67804 & 23.1 & 1.2 \\
56355.63611 & 33.8 & 1.3 \\
56357.65331 & 33.0 & 1.2 \\
56358.70107 & 39.4 & 2.4 \\
56428.46819 & -99.9 & 1.2 \\
56431.48616 & -105.3 & 1.7 \\
56434.49819 & -110.7 & 1.1 \\
56438.46472 & -119.0 & 1.1 \\
\hline & &
\end{tabular}

planet candidate are highly uncertain (e.g., Tinney et al. 2011; Wittenmyer et al. 2012a; Horner et al. 2012b) or when data are sparse (Wittenmyer et al. 2011). We allowed the genetic algorithm to search a wide parameter space and it ran for 50,000 iterations, testing a total of about $10^{7}$ possible configurations. We then fit the two datasets simultaneously using GaussFit (Jefferys et al. 1987), a generalized least-squares program used here to solve a Keplerian radial velocity orbit model. The GaussFit model has the ability to allow the offsets between multiple datasets to be a free parameter. The parameters of the best two-planet solution obtained by the genetic algorithm were used as initial inputs to GaussFit and a jitter of $3.3 \mathrm{~m} \mathrm{~s}^{-1}$ was added in quadrature to the uncertainty of each observation (following Tinney et al. 2006). The best-fit Keplerian solutions are given in Table 3; planetary minimum masses $m$ sin $i$ are derived using a stellar mass of $1.014 \pm 0.046 M_{\odot}$ (Takeda et al. 2007). This fit has a reduced $\chi^{2}$ of 1.63 and a total rms of $6.32 \mathrm{~m} \mathrm{~s}^{-1}$ (AAT-7.67 $\mathrm{m} \mathrm{s}^{-1}$; PFS-2.75 m s${ }^{-1}$ ).

\subsection{Dynamical Fit}

Because the two planets are massive enough and orbit close enough to each other to be interacting, we also apply a full dynamical model to these data. This model includes the effects of planet-planet interactions and can be used to place constraints on the system's inclination to the sky plane, $i$, a quantity that cannot be determined from Keplerian fitting alone. The system inclination then sets the true masses of the planets. The technique is described fully in Tan et al. (2013) for the HD 82943 twoplanet system. The Levenberg-Marquardt (Press et al. 1992) method is adopted as our fitting method. Using the Keplerian
Table 4

Dynamical Fit Solutions

\begin{tabular}{|c|c|c|}
\hline Parameter & Planet b & Planet c \\
\hline$K\left(\mathrm{~m} \mathrm{~s}^{-1}\right)$ & $85.4 \pm 2.3$ & $62.3 \pm 1.8$ \\
\hline Period (days) & $189.65 \pm 0.21$ & $376.93 \pm 0.69$ \\
\hline Eccentricity & $0.265 \pm 0.021$ & $0.198 \pm 0.029$ \\
\hline$\omega(\mathrm{deg})$ & $198.3 \pm 3.6$ & $294.5 \pm 11.3$ \\
\hline Mean anomaly (deg) & $105.0 \pm 5.0$ & $153.4 \pm 9.0$ \\
\hline$a(\mathrm{AU})$ & $0.65 \pm 0.01$ & $1.03 \pm 0.02$ \\
\hline$i(\operatorname{deg})$ & 90.0 & \\
\hline Mass $\left(M_{\text {Jup }}\right)$ & $2.35 \pm 0.12$ & $2.19 \pm 0.12$ \\
\hline$\chi_{v}^{2}$ & 1.70 & \\
\hline $\mathrm{rms}\left(\mathrm{m} \mathrm{s}^{-1}\right)$ & 6.54 & \\
\hline$K\left(\mathrm{~m} \mathrm{~s}^{-1}\right)$ & $83.0 \pm 2.1$ & $61.5 \pm 1.6$ \\
\hline Period (days) & $189.01 \pm 0.27$ & $379.32 \pm 0.92$ \\
\hline Eccentricity & $0.292 \pm 0.022$ & $0.244 \pm 0.026$ \\
\hline$\omega(\operatorname{deg})$ & $202.3 \pm 3.2$ & $285.3 \pm 10.6$ \\
\hline Mean anomaly (deg) & $102.8 \pm 3.9$ & $163.2 \pm 8.3$ \\
\hline$a(\mathrm{AU})$ & $0.65 \pm 0.01$ & $1.03 \pm 0.02$ \\
\hline$i(\mathrm{deg})$ & 40.2 & \\
\hline Mass $\left(M_{\text {Jup }}\right)$ & $3.50 \pm 0.17$ & $3.32 \pm 0.17$ \\
\hline$\chi_{v}^{2}$ & 1.72 & \\
\hline $\operatorname{rms}\left(\mathrm{m} \mathrm{s}^{-1}\right)$ & 6.59 & \\
\hline$K\left(\mathrm{~m} \mathrm{~s}^{-1}\right)$ & $81.4 \pm 2.3$ & $63.1 \pm 1.6$ \\
\hline Period (days) & $189.43 \pm 0.82$ & $378.29 \pm 2.81$ \\
\hline Eccentricity & $0.308 \pm 0.020$ & $0.293 \pm 0.021$ \\
\hline$\omega(\mathrm{deg})$ & $205.7 \pm 3.4$ & $284.3 \pm 9.9$ \\
\hline Mean anomaly (deg) & $99.5 \pm 4.0$ & $165.8 \pm 6.8$ \\
\hline$a(\mathrm{AU})$ & $0.649 \pm 0.012$ & $1.029 \pm 0.021$ \\
\hline$i(\operatorname{deg})$ & 20.8 & \\
\hline Mass $\left(M_{\text {Jup }}\right)$ & $6.22 \pm 0.33$ & $6.10 \pm 0.31$ \\
\hline$\chi_{v}^{2}$ & 1.80 & \\
\hline $\mathrm{rms}\left(\mathrm{m} \mathrm{s}^{-1}\right)$ & 6.76 & \\
\hline
\end{tabular}

best fit as an initial guess, assuming coplanar edge-on orbits, the Levenberg-Marquardt algorithm converges to a local minimum with a $\chi_{v}^{2}$ of about 1.70 and an rms of about $6.54 \mathrm{~m} \mathrm{~s}^{-1}$. Based on this local minimum, we conduct a parameter grid search (Lee et al. 2006; Tan et al. 2013) to ensure a global search for the best fit. This minimum is indeed a global dynamical best fit assuming coplanar edge-on orbits; two other local minima with slightly larger $\chi_{v}^{2}$ have been found. The coplanar edge-on best-fit parameters are listed in Table 4, with their error bars determined by the covariance matrix. This fit and its residuals are shown in the left panel of Figure 1. The right panel of Figure 1 shows that both resonance angles are librating, with amplitudes of $\pm 40^{\circ}$ $\left(\theta_{1}\right)$ and $\pm 60^{\circ}\left(\theta_{2}\right)$.

Assuming the planets are in coplanar orbits, we then allow the inclination to the sky plane to vary along with other fitting parameters. Figure 2 shows $\chi^{2}$ and $\mathrm{rms}$ as a function of $\sin i$ and Figure 3 shows best-fit parameters as a function of $\sin i$. The $\chi^{2}$ curve is shallow in the range of $\sin i \gtrsim 0.6$, but then shows a clear local minimum at $\sin i \sim 0.36(i=20.8)$. Two further local minima were found, at inclinations of $i=90.0$ and 40.2. The parameters of these three solutions are given in Table 4; the

Table 3

Keplerian Orbital Solutions

\begin{tabular}{lcccrr}
\hline \hline Planet & $\begin{array}{c}\text { Period } \\
\text { (days) }\end{array}$ & $\begin{array}{c}T_{0} \\
(\mathrm{JD}-2400000)\end{array}$ & $e$ & $\begin{array}{c}\omega \\
\text { (degrees) }\end{array}$ & $\begin{array}{c}\mathrm{K} \\
\left(\mathrm{m} \mathrm{s}^{-1}\right)\end{array}$ \\
\hline HD 73526 b & $188.9 \pm 0.1$ & $52856 \pm 2$ & $0.29 \pm 0.03$ & $196 \pm 5$ & $82.7 \pm 2.5$ \\
HD 73526 c & $379.1 \pm 0.5$ & $53300 \pm 10$ & $0.28 \pm 0.05$ & $272 \pm 10$ & $65.1 \pm 2.6$ \\
\hline
\end{tabular}



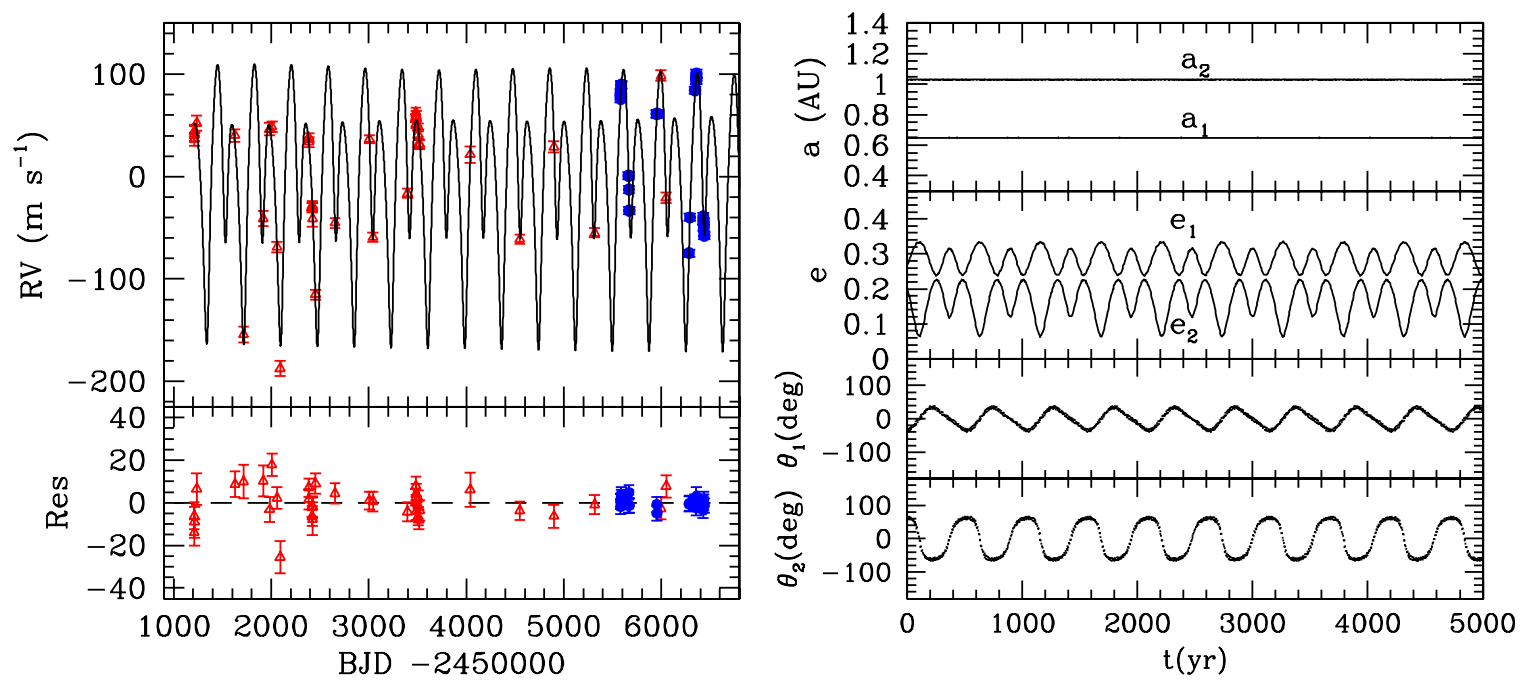

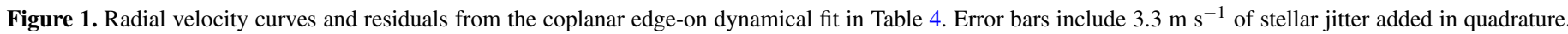

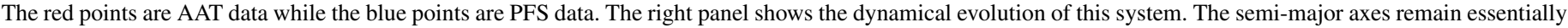
constant, while the eccentricities show secular variations on timescales of centuries. The fit is in a 2:1 MMR with both $\theta_{1}$ and $\theta_{2}$ librating around $0^{\circ}$.

(A color version of this figure is available in the online journal.)
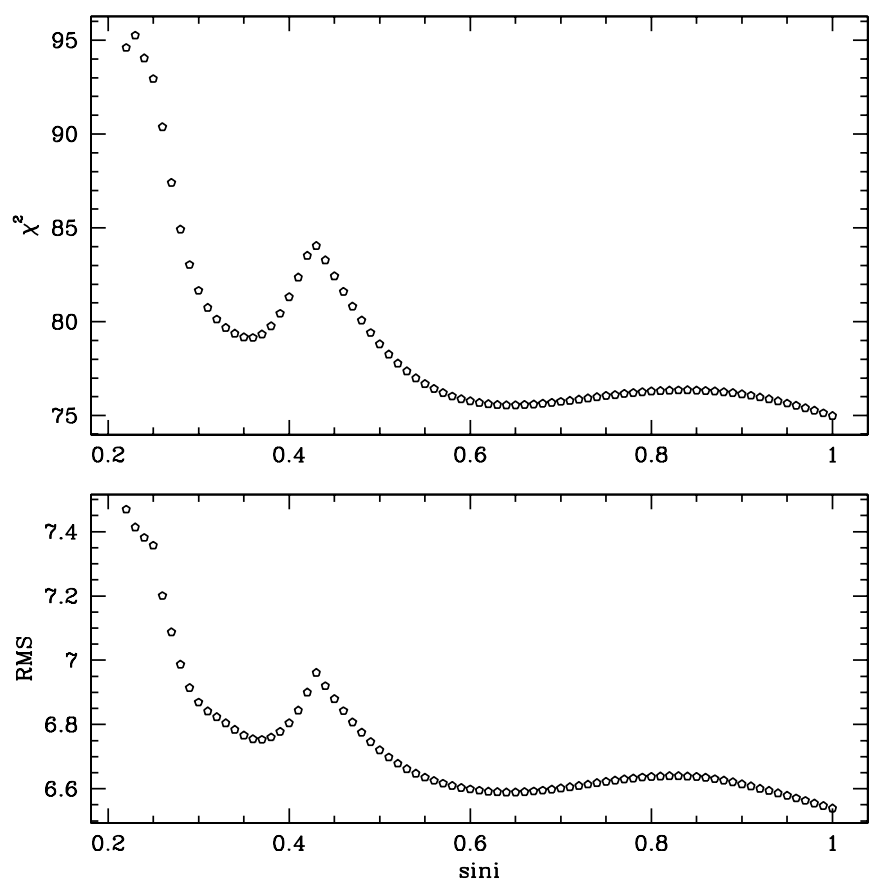

Figure 2. $\chi^{2}$ and rms as a function of $\sin i$.

planetary masses scale accordingly as $1 / \sin i$, resulting in more massive planets for the low-inclination solutions.

The $\chi^{2}$ curve and fitting parameters (Figure 3) show discontinuities along the $\sin i$ axis, especially those near $\sin i \sim 0.43$. To understand these discontinuities, we explore grids in different fixed $\sin i$, to see the evolution of the parameter space along different inclinations. Figure 4 shows $K_{1}-K_{2}$ grids for different $\sin i$. Initially, when the orbits are at $\sin i \sim 0.43$, there is only one $\chi^{2}$ minimum $\left(K_{1} \sim 84\right)$. Then a new local minimum $\left(K_{1} \sim 82\right)$ appears around $\sin i=0.427$, whose fitting parameters are significantly different from the original minimum $\left(K_{1} \sim 84\right)$. When $\sin i$ drops down to 0.425 , the original minimum vanishes and the new one becomes a single minimum in parameter space. The appearance of the additional $\chi_{v}^{2}$ minimum results in the big "jump" of fitting parameters at about $\sin i=0.43$ (see Figure 3).

In summary, we have four possible configurations for this system (one Keplerian fit and three dynamical fits). The four solutions are not substantially different from one another, apart from the $\sin i$ factor for the three solutions in Table 4, which serves to increase the planetary masses relative to the Keplerian scenario in which we have assumed the planets to be at their minimum masses ( $\mathrm{m} \sin i$ ). As a first-order check of dynamical stability, the best-fit system configuration at each inclination was integrated for $10^{8} \mathrm{yr}$. For all fits so tested, at inclinations of $26.7,30.0,33.4,36.9,40.0$, and $90.0(\sin i=0.45,0.5$, $0.55,0.6,0.64$, and 1.0 ), the systems remained stable for $10^{8} \mathrm{yr}$. However, since dynamical stability is highly dependent on the initial conditions, we expand on these tests in the next section to obtain a more robust and complete picture of the stability of the various configurations.

\section{DYNAMICAL STABILITY TESTING}

We have found four possible solutions for the HD 73526 system, which significantly differ in inclination (and hence in planetary masses). It is therefore critical to perform dynamical stability tests on these configurations, as the solution with the absolute $\chi^{2}$ minimum may prove dynamically unfeasible.

\subsection{Long-term Stability}

When analyzing any multiple-planet system, it is prudent to investigate the long-term dynamical stability of the system. As more multi-planet systems are discovered, the announcement of planetary systems that turn out to be dynamically unfeasible has become increasingly common. Detailed $N$-body simulations can be used to test the veracity of planet claims. Sometimes, the results of such tests have shown that some systems simply cannot exist in their proposed configuration on astronomically relevant timescales (e.g., Horner et al. 2011; Wittenmyer et al. 2012a; Horner et al. 2012a; Wittenmyer et al. 2013). In other cases, dynamical testing can place additional constraints on planetary 

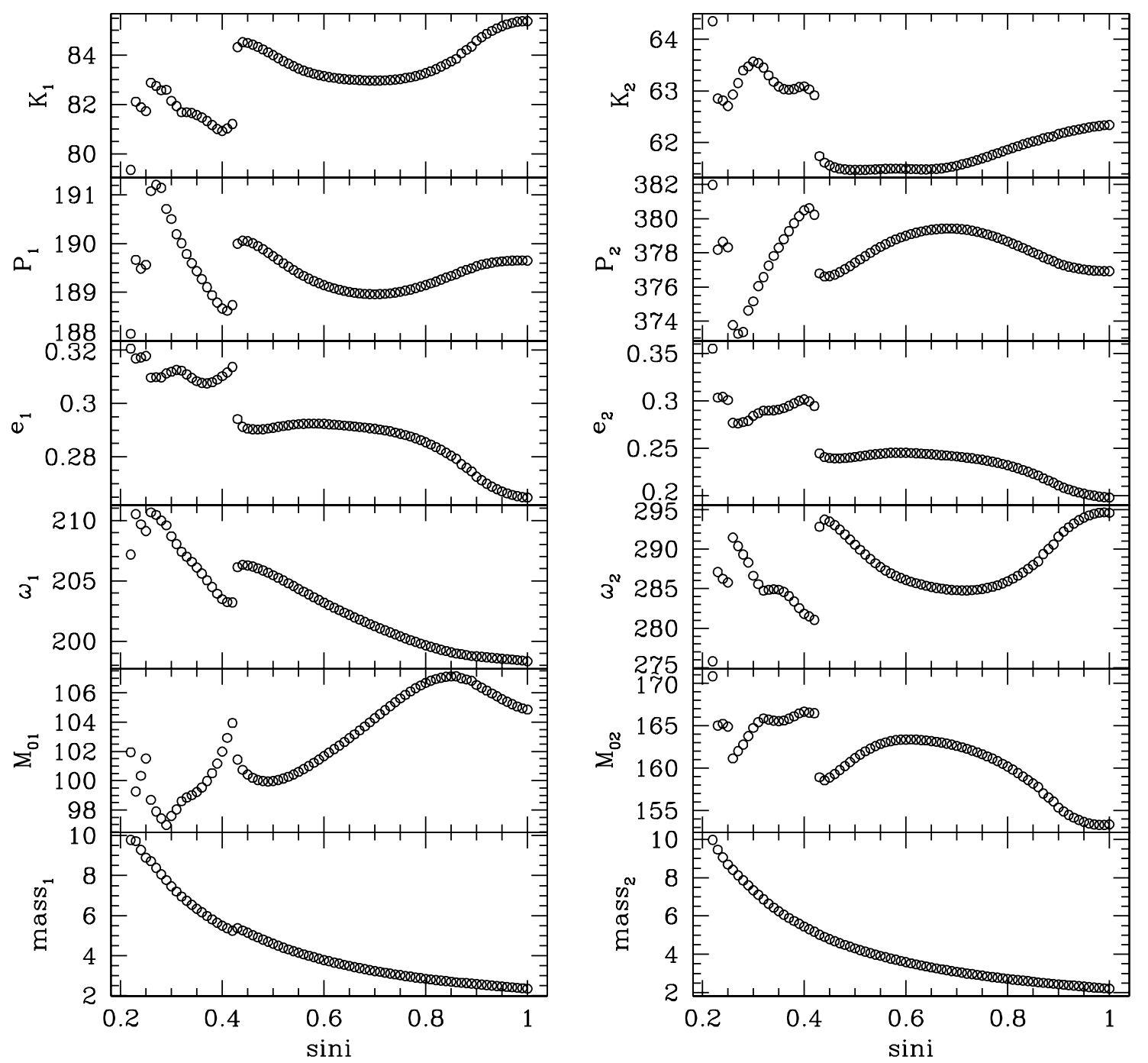

Figure 3. Best-fit parameters as a function of $\sin i$.

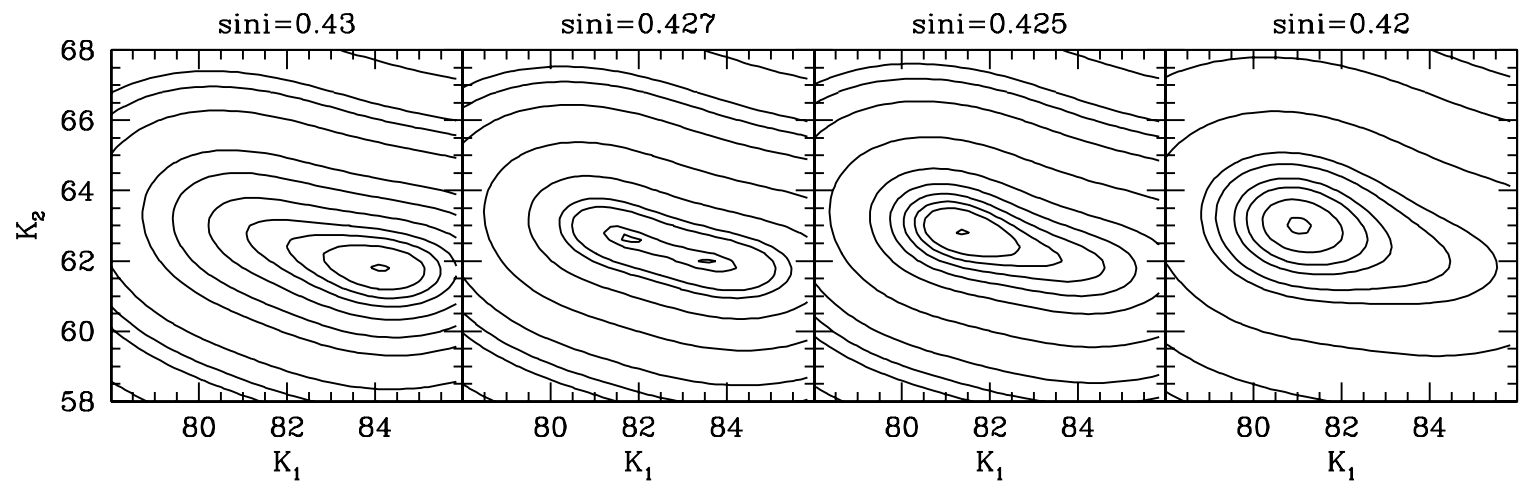

Figure 4. Evolution of $\chi_{v}^{2}$ contours in $K_{1}-K_{2}$ space as a function of $\sin i$.

systems, particularly when the planets are in or near resonances (e.g., Robertson et al. 2012a, 2012b; Wittenmyer et al. 2012b). In this section, we examine the various solutions for the HD 73526 system, performing detailed dynamical tests of the planetary system configurations as given in Tables 3 and 4 . Given that the four solutions are not substantially different from each other in terms of goodness of fit, these dynamical stability tests can serve to discern which scenario is most plausible; a solution that is favored by the fitting process may prove to be unstable, and hence unfeasible.

\subsection{Procedure}

As in our previous dynamical work (e.g., Marshall et al. 2010; Wittenmyer et al. 2012c; Horner et al. 2012b), we used the Hybrid integrator within the $N$-body dynamics package MERCURY (Chambers 1999) to perform our integrations. We held the initial orbit of the inner planet fixed at its best-fit parameters, as given in Table 3, and then created 126,075 test systems. In those test systems, the initial orbit of the outer planet was varied systematically in semi-major axis $a$, eccentricity $e$, 

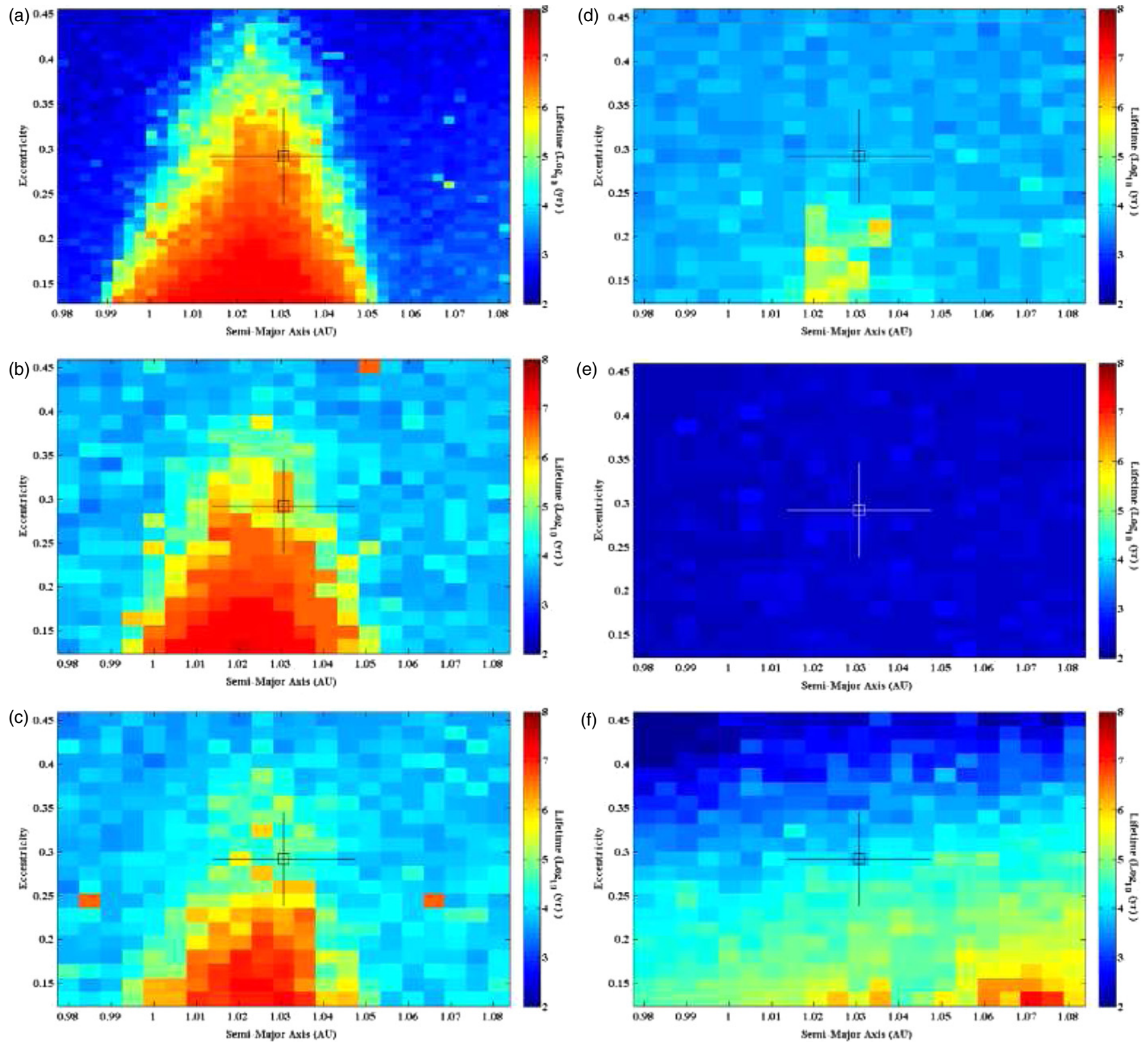

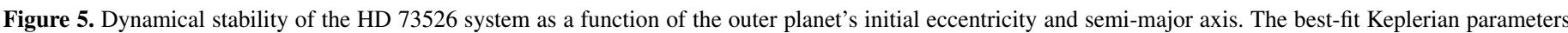

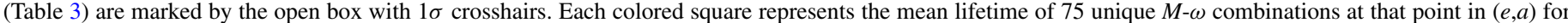
the outer planet. Panel (a) is the coplanar case and panels (b)-(f) are the mutually inclined scenarios, for inclinations of $5^{\circ}, 15^{\circ}, 45^{\circ}, 135^{\circ}$, and $180^{\circ}$, respectively. (A color version of this figure is available in the online journal.)

periastron argument $\omega$, and mean anomaly $M$, resulting in a $41 \times$ $41 \times 15 \times 5$ grid of "clones" spaced evenly across the $3 \sigma$ range in those parameters. We assumed the planets were coplanar with each other and, for the Keplerian case, we assigned masses equivalent to their minimum mass, $m \sin i$ (Table 3 ). We then followed the dynamical evolution of each test system for a period of $100 \mathrm{Myr}$ and recorded the times at which either of the planets was removed from the system. Planets were removed if they collided with one another, hit the central body, or reached a barycentric distance of $10 \mathrm{AU}$.

We performed these dynamical simulations for the Keplerian fit $\left(i=90^{\circ}\right)$, the dynamical fit at $i=90^{\circ}$, and the lowestinclination dynamical fit: the configuration given in Table 4 at $i=20.8$. For the last scenario, the planet masses were scaled according to the derived system inclination $i$. Clearly, the masses of the planets are a proxy for the expected dynamical stability-systems containing more massive planets are likely to be less stable. Hence, the three scenarios we have tested, at $i=90^{\circ}$ and $i=20.8$, represent the extremes of dynamical stability (or instability) for the HD 73526 system.

To explore the effects of mutual inclinations between the planets, we performed five additional $N$-body simulations, for scenarios in which the two planets were inclined with respect to each other. These simulations were set up exactly as described above, using the parameters of the Keplerian solution (Table 3), except at a lower resolution due to computational limitations: a $21 \times 21 \times 5 \times 5$ grid in $a, e, \omega$, and $M$. Five runs were performed, at mutual inclinations of $5^{\circ}, 15^{\circ}, 45^{\circ}, 135^{\circ}$, and $180^{\circ}$. The latter two cases represent retrograde scenarios, which can sometimes 


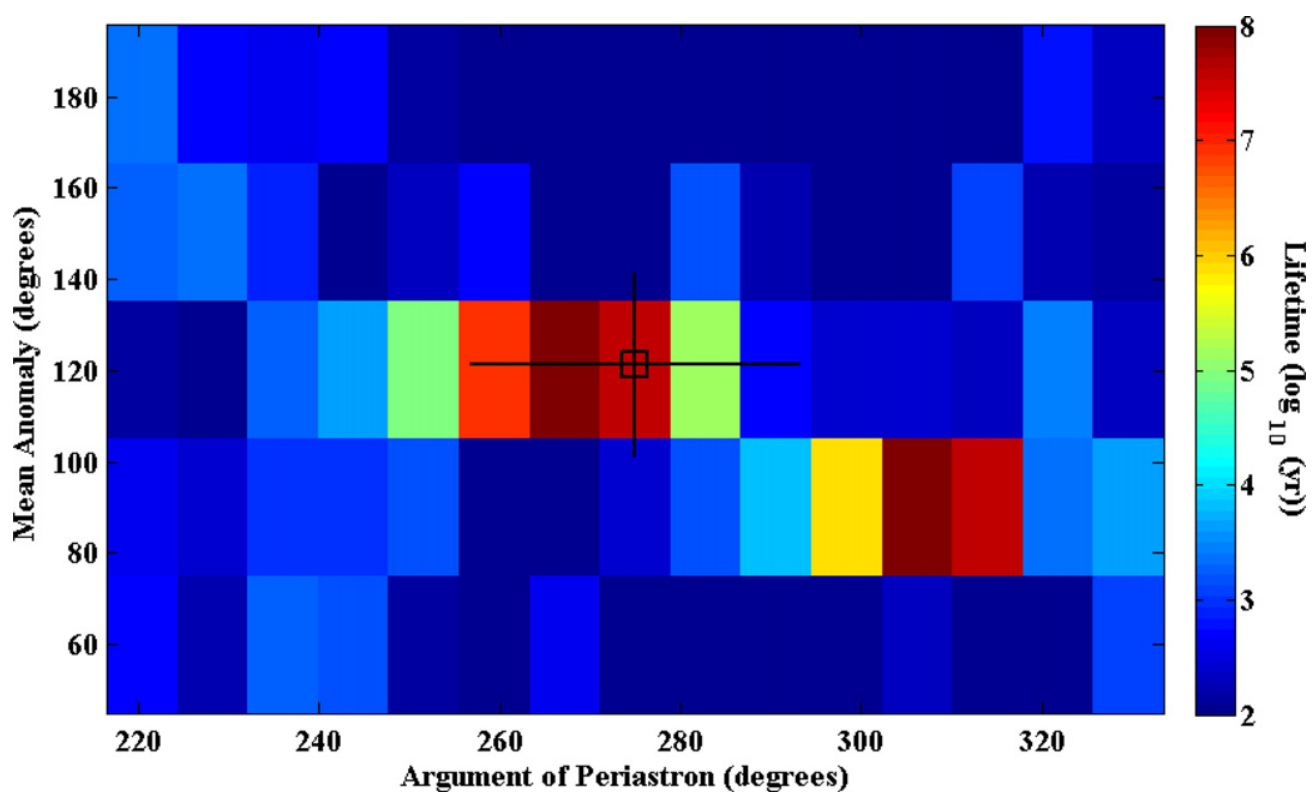

Figure 6. Dynamical stability of the best-fit Keplerian solution for the HD 73526 system for a $15 \times 5$ grid of $\omega$ and $M$. The semi-major axis and eccentricity have been fixed to their best-fit values. The colors and symbols have the same meaning as in Figure 5; this plot shows results from the 75 individual simulations that were averaged in the center colored square of Figure 5. The best-fit solution lies squarely in the most stable region of this subset of simulations.

(A color version of this figure is available in the online journal.)
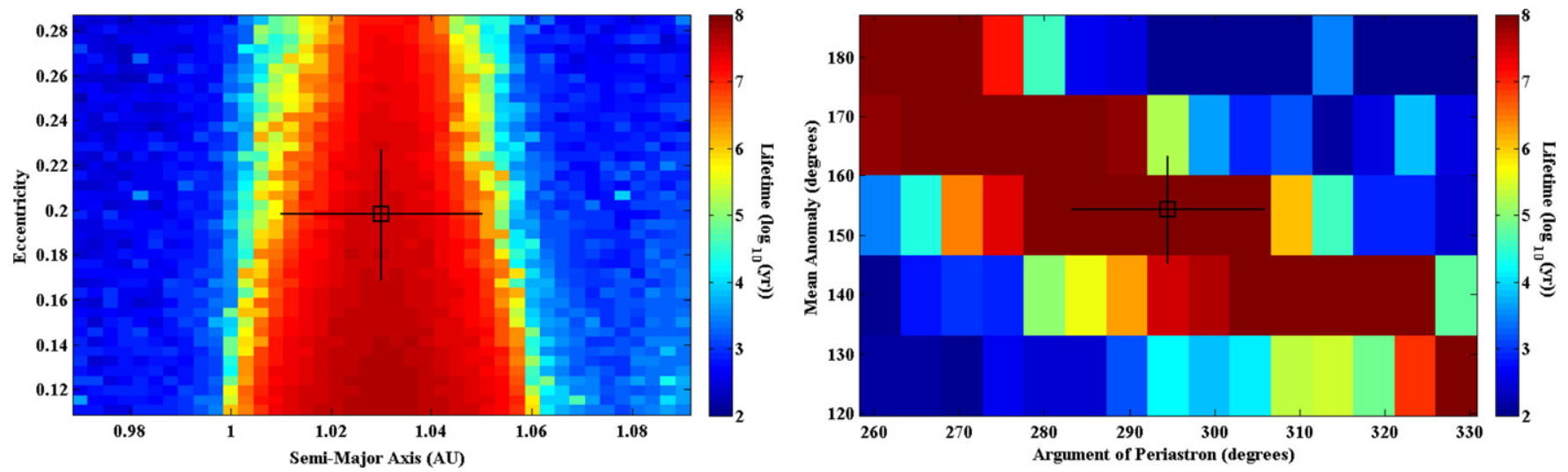

Figure 7. Left: stability of the HD 73526 system as a function of the outer planet's initial eccentricity and semi-major axis. The colors and symbols have the same meaning as in Figure 5. For this system, we used the $i=90^{\circ}$ solution (Table 4). As compared with the Keplerian solution, this fit results in substantially enhanced stability throughout the $1 \sigma$ range. Right: same as Figure 6, but for the dynamical-fit $i=90^{\circ}$ solution.

(A color version of this figure is available in the online journal.)

allow for a larger range of dynamically stable orbits (Eberle \& Cuntz 2010; Horner et al. 2011).

\subsection{Results}

The results of our dynamical stability simulations for the Keplerian solution are shown in Figure 5. We show six panels, for the coplanar and five mutually inclined scenarios as described above. For the coplanar and $5^{\circ}$ cases (panels (a) and (b)), the best-fit set of parameters (shown by the open square with $1 \sigma$ crosshairs) lies in a region of moderate stability, with mean system survival times of $\sim 10^{6} \mathrm{yr}$. The stability rapidly degrades as the inclination between the planets becomes significant and even for retrograde cases (panels (e) and (f)), the nominal bestfit system destabilizes within $10^{4}$ yr. From these simulations, we can conclude that the HD 73526 planets are most likely coplanar with each other. Panels (a) and (b) also show that the stability of the system increases as the outer planet takes on lower eccentricities. For $e \lesssim 0.2$, the mean survival times exceed $10^{7} \mathrm{yr}$. This is not a surprising result, as high eccentricities generally increase the possibility of strong interactions or even orbit crossings although systems in protected resonances may remain stable for some values of $M$ and $\omega$. Indeed, the statistics of multi-planet systems show that planets in multiple systems tend to have lower eccentricities (Wright et al. 2009; Wittenmyer et al. 2009).

As shown in Figure 5, the Keplerian best-fit solution is stable on Myr timescales. However, the colored squares in Figure 5 represent the mean survival times across the range of mean anomalies and $\omega$ tested. As the best-fit solution for the HD 73526 system places the planets on resonant orbits, their stability will naturally be highly sensitive to the values of these angles. Hence, Figure 6 shows the outcomes of the 75 individual simulations performed at the best-fit $a$ and $e$ spanning a $5 \times 15$ grid in $M$ and $\omega$. We see that the nominal solution (where $a$ and $e$ are fixed at the best-fit values) lies at the point of maximum stability. While this is reassuringly consistent with our expectations of enhanced stability within the resonance, we caution that each colored square in Figure 6 represents only a single run and 




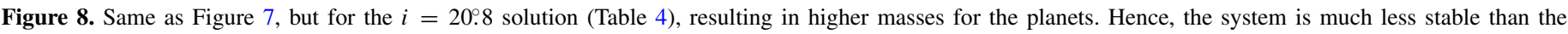
minimum-mass case explored in Figure 7, with mean survival times of the order of $1000 \mathrm{yr}$.

(A color version of this figure is available in the online journal.)

dynamical evolution is known to be a chaotic process (e.g., Horner et al. 2004a, 2004b).

The long-term stability results for the dynamical fit with $i=90^{\circ}$ are shown in Figure 7. It is immediately apparent that this solution results in a higher degree of stability, with the entire $1 \sigma$ region exhibiting mean lifetimes exceeding $10^{7} \mathrm{yr}$. The right panel of Figure 7 shows the results from the 75 individual runs in the central best-fit square, as in Figure 6. For this case, when we examine the dependence on $M$ and $\omega$, we see that the entire diagonal region (including the best fit) is stable for $10^{8}$ yr. In contrast, Figure 8 shows the dynamical stability of the $i=20.8$ solution from Table 4 . Although this fit is formally almost as good as the Keplerian fit, the increased masses implied by the inclination render the system unstable on short timescales (1000 yr).

\section{DISCUSSION AND CONCLUSIONS}

We have fit the HD 73526 system using both kinematic and dynamical techniques, yielding four possible solutions. There are no compelling differences among the four models in terms of their goodness-of-fit statistics or derived planetary parameters. The only significant distinguishing characteristics are the planet masses derived from the system inclinations in the dynamical fits (Table 4). We thus turned to a detailed dynamical stability mapping procedure in which we tested a broad range of parameters about the best-fit solutions.

Our dynamical stability testing showed that the Keplerian model yielded a system that was stable on Myr timescales, with stability increasing for lower eccentricities (Figure 5). The interacting dynamical fitting procedure gave three "best" solutions, one of which was at a system inclination of $90^{\circ}$ (giving planet masses equal to the $\mathrm{m}$ sin $i$ minimum masses used in the Keplerian model). Our stability testing for the inclined solution at $i=20.8$ resulted in severe instabilities throughout the allowed $3 \sigma$ parameter space. The increased planetary masses for the low-inclination solutions appear to destabilize the system on astronomically short timescales $(<1000 \mathrm{yr})$. This result leads us to reject the $i=20.8$ scenario. While the individual best-fit solutions proved stable for $i>26.7$ (as noted in Section 3.2), it is clear that the region of long-term stability expands as the system inclination increases. We thus adopt the $i=90^{\circ}$ dynamical fit for two primary reasons: first, the planets are massive enough that they are certainly interacting with each other, as evidenced by the 2:1 resonant configuration, and second, this fit proved to be significantly more stable than the Keplerian fit (Figure 5). We note in passing that if the system's inclination is indeed near $90^{\circ}$, there is the possibility that one or both planets transit.

This work has shown how dynamical stability considerations can serve to constrain the configuration of a planetary system when the $\chi^{2}$ surface is such that a clear minimum is not evident (e.g., Campanella 2011). We have combined two fitting methods with the detailed dynamical simulations to present an updated view of the interesting 2:1 resonant planetary system orbiting HD 73526.

This research has made use of NASA's Astrophysics Data System (ADS) and the SIMBAD database, operated at CDS, Strasbourg, France. This research has also made use of the Exoplanet Orbit Database and the Exoplanet Data Explorer at exoplanets.org (Wright et al. 2011). M.H.L. was supported in part by the Hong Kong RGC grant HKU 7024/13P. D.M. acknowledges funding from the BASAL CATA Center for Astrophysics and Associated Technologies PFB-06, the The Milky Way Millennium Nucleus from the Ministry for the Economy, Development, and Tourism's Programa Iniciativa Científica Milenio P07-021F. C.G.T. and J.H. are supported by Australian Research Council grants DP0774000 and DP130102695. Australian access to the Magellan Telescopes was supported through the National Collaborative Research Infrastructure Strategy of the Australian Federal Government.

\section{REFERENCES}

Albrecht, S., Winn, J. N., Butler, R. P., et al. 2012, ApJ, 744, 189 Albrecht, S., Winn, J. N., Johnson, J. A., et al. 2011, ApJ, 738, 50 Anglada-Escudé, G., Arriagada, P., Vogt, S. S., et al. 2012, ApJL, 751, L16 
Arriagada, P., Anglada-Escudé, G., Butler, R. P., et al. 2013, ApJ, 771, 42 Baluev, R. V. 2011, CeMDA, 111, 235

Batalha, N. M., Rowe, J. F., Bryson, S. T., et al. 2013, ApJS, 204, 24

Beaugé, C., Ferraz-Mello, S., \& Michtchenko, T. A. 2003, ApJ, 593, 1124

Beaugé, C., Michtchenko, T. A., \& Ferraz-Mello, S. 2006, MNRAS, 365, 1160

Borucki, W. J., Koch, D., Basri, G., et al. 2010, Sci, 327, 977

Butler, R. P., Marcy, G. W., Williams, E., et al. 1996, PASP, 108, 500

Campanella, G. 2011, MNRAS, 418, 1028

Chambers, J. E. 1999, MNRAS, 304, 793

Cochran, W. D., Endl, M., Wittenmyer, R. A., \& Bean, J. L. 2007, ApJ, 665,1407

Crane, J. D., Shectman, S. A., \& Butler, R. P. 2006, Proc. SPIE, 6269, 96

Crane, J. D., Shectman, S. A., Butler, R. P., Thompson, I. B., \& Burley, G. S. 2008, Proc. SPIE, 7014, 238

Crane, J. D., Shectman, S. A., Butler, R. P., et al. 2010, Proc. SPIE, 7735, 170

Diego, F., Charalambous, A., Fish, A. C., \& Walker, D. D. 1990, Proc. SPIE, 1235,562

Eberle, J., \& Cuntz, M. 2010, ApJL, 721, L168

Fabrycky, D. C., Lissauer, J. J., Ragozzine, D., et al. 2012, arXiv:1202.6328

Ferraz-Mello, S., Beaugé, C., \& Michtchenko, T. A. 2003, CeMDA, 87, 99

Horner, J., Evans, N. W., \& Bailey, M. E. 2004a, MNRAS, 354, 798

Horner, J., Evans, N. W., \& Bailey, M. E. 2004b, MNRAS, 355, 321

Horner, J., Hinse, T. C., Wittenmyer, R. A., Marshall, J. P., \& Tinney, C. G. 2012a, MNRAS, 427, 2812

Horner, J., Marshall, J. P., Wittenmyer, R. A., \& Tinney, C. G. 2011, MNRAS, 416, L11

Horner, J., Wittenmyer, R. A., Hinse, T. C., \& Tinney, C. G. 2012b, MNRAS, 425,749

Jefferys, W. H., Fitzpatrick, M. J., \& McArthur, B. E. 1987, CMec, 41, 39

Johnson, J. A., Payne, M., Howard, A. W., et al. 2011, AJ, 141, 16

Kennedy, G. M., Wyatt, M. C., Bryden, G., Wittenmyer, R., \& Sibthorpe, B. 2013, MNRAS, 436, 898

Laughlin, G. 2000, ApJ, 545, 1064

Lee, M. H. 2004, ApJ, 611, 517

Lee, M. H., Butler, R. P., Fischer, D. A., Marcy, G. W., \& Vogt, S. S. 2006, ApJ, 641,1178

Lee, M. H., Fabrycky, D., \& Lin, D. N. C. 2013, ApJ, 774, 52

Lissauer, J. J., Ragozzine, D., Fabrycky, D. C., et al. 2011, ApJS, 197, 8
Lithwick, Y., \& Wu, Y. 2012, ApJL, 756, L11

Marcy, G. W., Butler, R. P., Fischer, D., et al. 2001, ApJ, 556, 296

Marshall, J., Horner, J., \& Carter, A. 2010, IJAsB, 9, 259

Marti, J. G., Giuppone, C. A., \& Beauge, C. 2013, MNRAS, 433, 928

Mayor, M., Udry, S., Naef, D., et al. 2004, A\&A, 415, 391

Michtchenko, T. A., Beaugé, C., \& Ferraz-Mello, S. 2008, MNRAS, 391, 215

Press, W. H., Teukolsky, S. A., Vetterling, W. T., \& Flannery, B. P. 1992, Numerical Recipes in Fortran: The Art of Scientific Computing (2nd ed; Cambridge: Cambridge Univ. Press)

Raymond, S. N., Barnes, R., Armitage, P. J., \& Gorelick, N. 2008, ApJL, 687, L107

Rivera, E. J., Laughlin, G., Butler, R. P., et al. 2010, ApJ, 719, 890

Robertson, P., Endl, M., Cochran, W. D., et al. 2012a, ApJ, 749, 39

Robertson, P., Horner, J., Wittenmyer, R. A., et al. 2012b, ApJ, 754, 50

Sándor, Z., Kley, W., \& Klagyivik, P. 2007, A\&A, 472, 981

Steffen, J. H., Fabrycky, D. C., Agol, E., et al. 2013, MNRAS, 428, 1077

Takeda, G., Ford, E. B., Sills, A., et al. 2007, ApJS, 168, 297

Tan, X., Payne, M. J., Lee, M. H., et al. 2013, ApJ, 777, 101

Tinney, C. G., Butler, R. P., Marcy, G. W., et al. 2003, ApJ, 587, 423

Tinney, C. G., Butler, R. P., Marcy, G. W., et al. 2006, ApJ, 647, 594

Tinney, C. G., Wittenmyer, R. A., Butler, R. P., et al. 2011, ApJ, 732, 31

Valenti, J. A., Butler, R. P., \& Marcy, G. W. 1995, PASP, 107, 966

Valenti, J. A., \& Fischer, D. A. 2005, ApJS, 159, 141

Veras, D., \& Ford, E. B. 2012, MNRAS, 420, L23

Vogt, S. S., Butler, R. P., Marcy, G. W., et al. 2005, ApJ, 632, 638

Wittenmyer, R. A., Endl, M., Cochran, W. D., Levison, H. F., \& Henry, G. W. 2009, ApJS, 182, 97

Wittenmyer, R. A., Endl, M., Wang, L., et al. 2011, ApJ, 743, 184

Wittenmyer, R. A., Horner, J., \& Marshall, J. P. 2013, MNRAS, 431, 2150

Wittenmyer, R. A., Horner, J., Marshall, J. P., Butters, O. W., \& Tinney, C. G. 2012a, MNRAS, 419, 3258

Wittenmyer, R. A., Horner, J., \& Tinney, C. G. 2012b, ApJ, 761, 165

Wittenmyer, R. A., Horner, J., Tuomi, M., et al. 2012c, ApJ, 753, 169

Wright, J. T., Fakhouri, O., Marcy, G. W., et al. 2011, PASP, 123, 412

Wright, J. T., Upadhyay, S., Marcy, G. W., et al. 2009, ApJ, 693, 1084

Wright, J. T., Veras, D., Ford, E. B., et al. 2011, ApJ, 730, 93

Zhang, N., Ji, J., \& Sun, Z. 2010, MNRAS, 405, 2016 